\title{
Mining Both Associated and Correlated Patterns
}

\author{
Zhongmei Zhou ${ }^{1,2}$, Zhaohui Wu ${ }^{1}$, Chunshan Wang ${ }^{1}$, and Yi Feng ${ }^{1}$ \\ ${ }^{1}$ College of Computer Science and Technology, Zhejiang University, China \\ ${ }^{2}$ Department of Computer Science, Zhangzhou Teacher's College, China \\ $\{z z m$, wzh, cswang, fengyi $\}$ aju.edu.cn
}

\begin{abstract}
Association mining cannot find such type of patterns, "the conditional probability that a customer purchasing $A$ is likely to also purchase $B$ is not only greater than the given threshold, but also much greater than the probability that a customer purchases only $B$. In other words, the sale of $A$ can increase the likelihood of the sale of $B$." Such kind of patterns are both associated and correlated. Therefore, in this paper, we combine association with correlation in the mining process to discover both associated and correlated patterns. A new interesting measure corr-confidence is proposed for rationally evaluating the correlation relationships. This measure not only has proper bounds for effectively evaluating the correlation degree of patterns, but also is suitable for mining long patterns. Our experimental results show that the mining combined association with correlation is quite a valid approach to discovering both associated and correlated patterns.
\end{abstract}

\section{Introduction}

Data mining is defined as the process of discovering significant and potentially useful patterns in large volume of data. Although association or correlation mining can find many interesting patterns, the following two kinds of patterns generated from only association or correlation mining are misleading or meaningless, because neither of the two cases can lead to the result that the sale of $A$ increases the likelihood of the sale of $B$.

1. $A$ and $B$ are associated but not correlated, that is, although the conditional probability that a customer purchasing $A$ is likely to also purchase $B$ is greater than the given threshold, the probability that a customer purchases only $B$ is not significantly less the conditional probability.

2. $A$ and $B$ are correlated but not associated, that is, although whether a customer purchases $B$ is significantly influenced by whether she/he purchases $A$, the probability that a customer purchases only $B$ is much greater than the conditional probability that a customer purchasing $A$ is likely to also purchase $B$.

If pattern $A B$ satisfies the following two conditions, then the sale of $A$ can increase the likelihood of the sale of $B$.

1. The conditional probability that a customer purchasing $A$ is likely to also purchase $B$ is great enough.

2. The probability that a customer purchases only $B$ is significantly less than the conditional probability that a customer purchasing $A$ is likely to also purchase $B$. 
For example, if $\mathrm{P}(\mathrm{B})=88 \%$ and $\mathrm{P}(\mathrm{B} / \mathrm{A})=90 \%$, then the sale of $A$ cannot increase the likelihood of the sale of $B$, even though the conditional probability $P(B / A)=90 \%$ is much greater than the given threshold. In this case, $A$ and $B$ are associated but not correlated. If $\mathrm{P}(\mathrm{B})=90 \%$ and $\mathrm{P}(\mathrm{B} / \mathrm{A})=20 \%$, then the sale of $A$ cannot increase the likelihood of the sale of $B$, even if the purchase of $B$ is influenced by the purchase of $A$. It is the case that $A$ and $B$ are correlated but not associated.

Patterns which satisfy the first condition are associated and patterns which satisfy the second condition are correlated, so a pattern which satisfies the two conditions is both associated and correlated. Therefore, in this paper, we combine association with correlation in the mining process to discover both associated and correlated patterns.

One difficulty is how to select a proper interestingness measure that can effectively evaluate the association degree of patterns, as there is still no universally accepted best measure for judging interesting patterns [6]. Omiccinski [5] introduced three alternative interestingness measures, called any-confidence, all-confidence and bond for mining associations. Won-young kim [9] and Young-koo lee [10] used allconfidence to discover interesting patterns although both of them defined a pattern which satisfies the given minimum all-confidence as a correlated pattern. Allconfidence can be computed efficiently using its downward closure property [5], so it is employed for association mining in this paper.

Another difficulty is that there are few measures which not only have proper bounds for effectively evaluating the correlation degree of patterns but also are suitable for mining long correlated patterns. The most commonly employed method for correlation mining is that of two-dimensional contingency table analysis of categorical data using the chi-square statistic as a measure of significance. Brin et al. [2] analyzed contingency tables to generate correlation rules that identify statistical correlation in both the presence and absence of items in patterns. H. Liu et al. [3] analyzed contingency tables to discover unexpected and interesting patterns that have a low lever of support and a high level of confidence. Bing Liu et al. [1] used contingency tables for pruning and summarizing the discovered correlations etc. Although the low chi-squared value (less than the cutoff value, e.g. 3.84 at the $95 \%$ significance lever [4]) efficiently indicates that all patterns $A B, \bar{A} B, A \bar{B}, \overline{A B}$ are independent, that is, $A$ and $B, \bar{A}$ and $B, A$ and $\bar{B}, \bar{A}$ and $\bar{B}$ are all independent. The high chi-squared value only indicates that at least one of patterns $A B, \overline{A B}, A \bar{B}, \overline{A B}$ is dependent, so it is possible that $A B$ is independent, i.e. $A$ and $B$ are independent, in spite of the high chi-squared value as showed in experimental results. Therefore, when only the presence of items is considered, in other words, when only the sale of $A$ and $B$ is concerned, the chi-squared value is not reasonable for measuring the dependence degree of $A$ and $B$. For other commonly used measures, the measure $P(A B) / P(A) P(B)$ [2] does not have proper bounds. $P(A B)-P(A) P(B) / \sqrt{P(A) P(B)(1-P(A))(1-P(B))}$ [8] is not suitable for generating long patterns. $P(A B)-P(A) P(B)$ [7] is not rational when $P(A B)$ is compared with $P(A) P(B)$. For example, if $P(A B)=0.02, P(A) P(B)=0.01$, $P\left(A^{\prime} B^{\prime}\right)=0.99$ and $P\left(A^{\prime}\right) P\left(B^{\prime}\right)=0.98$, then $P(A B)-P(A) P(B)=P\left(A^{\prime} B^{\prime}\right)-P\left(A^{\prime}\right) P\left(B^{\prime}\right)$. The correlation degree of $A$ and $B$ is equal to the correlation degree of $A^{\prime}$ and $B^{\prime}$ by $P(A B)-P(A) P(B)$. However $P(A B) / P(A) P(B)=2$ and $P\left(A^{\prime} B^{\prime}\right) / P\left(A^{\prime}\right) P\left(B^{\prime}\right)=1.01$, the 
correlation degree of $A$ and $B$ is evidently higher than the correlation degree of $A^{\prime}$ and $B^{\prime}$. In this paper, a new interestingness measure corr-confidence is proposed for correlation mining. This measure not only has proper bounds for effectively evaluating the correlation degree of patterns, but also is suitable for mining long patterns.

The remainder of this paper is organized as follows: In section 2, some related concepts are given and an algorithm is developed for discovering both associatedcorrelated patterns. We report our experimental and performance results in section 3 . Section 4 concludes the paper.

\section{Mining Both Associated and Correlated Patterns}

This section first formalizes some related concepts and then gives an algorithm for efficiently discovering all both associated and correlated patterns.

In statistical theory, $A_{1}, A_{2}, \cdots, A_{n}$ are independent if $\forall k$ and $\forall 1 \leq i_{1}<i_{2}<\cdots<i_{k} \leq n$,

$$
P\left(A_{i_{1}} A_{i_{2}} \quad \cdots \quad A_{i_{k}}\right)=P\left(A_{i_{1}}\right) P\left(A_{i_{2}}\right) \cdots P\left(A_{i_{k}}\right)
$$

In this paper, let all patterns have more than one item. A new measure corrconfidence (denoted as $\rho$ ) is given as follows using (1):

1. If a pattern has two items, such as pattern $A B$, then

$$
\rho(A B)=P(A B)-P(A) P(B) / P(A B)+P(A) P(B)
$$

2. If a pattern has more than two items, such as pattern $X=\left\{i_{1} i_{2} \cdots i_{n}\right\}$, then

$$
\rho(X)=\frac{P\left(i_{1} i_{2}, \cdots, i_{n}\right)-P\left(i_{1}\right) P\left(i_{2}\right) \cdots P\left(i_{n}\right)}{P\left(i_{1} i_{2}, \cdots, i_{n}\right)+P\left(i_{1}\right) P\left(i_{2}\right) \cdots P\left(i_{n}\right)}, \quad(n \geq 1) .
$$

From (2) and (3), we can see that $\rho$ has two bounds, i.e. $-1 \leq \rho \leq 1$.

Let $\eta$ be a given minimum corr-confidence, if pattern $X$ has two items $A, B$ and $|\rho(A B)|>\eta$, then $X$ is called a correlated pattern or $A$ and $B$ are called correlated, else $A$ and $B$ are called independent. If pattern $X$ has more than two items, we define a correlated pattern and an independent pattern as follows:

Definition 1 (a correlated pattern). Pattern $X$ is called a correlated pattern, if and only if there exists a pattern $Y$ which satisfies $Y \subseteq X$ and $|\rho(Y)|>\eta$.

Definition 2 (an independent pattern). If pattern $X$ is not a correlated pattern, then it is called an independent pattern.

By the definition 1, we conclude that (1) if pattern $X$ is a correlated pattern, any super pattern of $X$ is a correlated pattern and pattern $X$ must have a subset which is a correlated pattern. (2) pattern $X$ must have two subsets $A$ and $B$ which are correlated. 
We define an associated pattern using the measure all-confidence [5].

Let $T=\left\{i_{1}, i_{2}, \cdots, i_{m}\right\}$ be a set of $m$ distinct literals called items and $D$ be a set of variable length transactions over $T$. Each transaction contains a set of items, $\left\{i_{j_{1}}, i_{j_{2}}, \cdots, i_{j_{k}}\right\} \subset T$. A transaction also has an associated unique identifier. Pattern $X$ is a subset of $T$. Let $p(X)$ be a power set of pattern $X$. The interestingness measure all-confidence (denoted as $\alpha$ ) of pattern $X$ is defined as follows [5]:

$$
\alpha=\frac{|\{d \mid d \in D \wedge X \subset d\}|}{\operatorname{MAX}\{i \mid \forall l(l \in p(X) \wedge l \neq \phi \wedge l \neq X \wedge i=|\{d \mid d \in D \wedge l \subset d\}|)\}}
$$

Definition 3 (an associated pattern). A pattern is called an associated pattern, if its all-confidence is greater than or equal to the given minimum all-confidence.

Definition 4 (an associated-correlated pattern). A pattern is called an associatedcorrelated pattern if it is not only an associated pattern but also a correlated pattern.

Let pattern $X$ be an associated-correlated pattern, then it must have two subsets $A$ and $B$ which satisfy the condition that the sale of $A$ can increase the likelihood of the sale of $B$.

Example 1. For the transaction database $T D B$ in Table 1 , we have $\alpha(A C)=2 / 3$ and $\alpha(C E)=2 / 3$, so both $A C$ and $C E$ have all-confidence $2 / 3$. We also have

$$
\rho(A C)=\frac{P(A C)-P(A) P(C)}{P(A C)+P(A) P(C)}=\frac{1}{4} \quad \text { and } \quad \rho(C E)=\frac{P(C E)-P(C) P(E)}{P(C E)+P(C) P(E)}=\frac{1}{19} .
$$

Let the given minimum all-confidence be 0.5 and the given minimum corrconfidence be 0.1 , then both $A C$ and $C E$ are associated patterns. However, pattern $A C$ is a correlated pattern and pattern $C E$ is an independent pattern. Therefore pattern $A C$ is an associated-correlated pattern and pattern $C E$ is an associated but not correlated pattern. From $P(A / C)=2 / 3, P(A)=2 / 5$, we can see that the sale of $C$ can increase the likelihood of the sale of $A$. Meanwhile, $P(C / A)=1, P(C)=3 / 5$, we can also see that the sale of $A$ can also increase the likelihood of the sale of $C$. However $P(C / E)=2 / 3$, and $P(C)=3 / 5$, the sale of $E$ cannot evidently increase the likelihood of the sale of $C$.

Table 1. Transaction database $T D B$

\begin{tabular}{|c|c|}
\hline Transaction id & Items \\
\hline 10 & $\mathrm{~A}, \mathrm{~B}, \mathrm{C}$ \\
\hline 20 & $\mathrm{C}, \mathrm{D}, \mathrm{E}$ \\
\hline 30 & $\mathrm{~A}, \mathrm{C}, \mathrm{D}, \mathrm{E}$ \\
\hline 40 & $\mathrm{D}, \mathrm{E}$ \\
\hline 50 & $\mathrm{~B}, \mathrm{D}$ \\
\hline
\end{tabular}


We mine all frequent associated-correlated patterns in two steps. First, we discover all frequent associated patterns, and then test whether they are correlated. We use a level-wise algorithm for discovering all frequent associated-correlated patterns.

\section{Algorithm}

Input a transaction database $T D B$, a minimum support $\xi$, a minimum corr-confidence $\eta$ and a minimum all-confidence $\lambda$.

Output the complete set of frequent associated-correlated patterns.

$c_{k}$ : Candidate patterns of size $k$

$L_{k}$ : Frequent associated patterns of size $k$

$I_{k}$ : Frequent associated and independent patterns of sizek

$I_{k}^{c}$ : Frequent associated-correlated patterns of size $k$

$L_{1}=\{$ frequent items

$I_{1} \leftarrow L_{1} ; I_{1}^{c} \leftarrow \phi$

For $\left(k=1 ; L_{k} ! \neq \phi ; k++\right)$ do begin

$C_{k+1}=$ candidates generated from $I_{k} * I_{k}$

$C_{k+1}^{\prime}=$ candidates generated from $I_{k} * I_{k}^{c}, I_{k}^{c} * I_{k}^{c}$

For each transaction $t$ in database do

increment the count of all candidates in $C_{k+1}, C_{k+1}^{\prime}$

that are contained in $t$

$L_{k+1}=$ candidates in $C_{k+1}$ with minimum support and minimum al1-confidence

$L_{k+1}^{\prime}=$ candidates in $C_{k+1}^{\prime}$ with minimum support and minimum all-confidence

$I_{k+1}^{c} \leftarrow L_{k+1}^{\prime}$

For each pattern $l_{k+1}$ in $L_{k+1}$

If $\left|\rho\left(l_{k+1}\right)\right|<\eta$ insert $l_{k+1}$ into $I_{k+1}$

Else insert $l_{k+1}$ into $I_{k+1}^{c}$

Return $\cup I_{k}^{c}$

Remark: In the algorithm, the prune step is performed as follows:

Forall patterns $c \in C_{k+1}$ do

Forall $k$-subsets $s$ of $c$ do

If $\left(s \notin L_{k}\right)$ or $\left(s \notin L_{k}\right)$ delete $c$ from $C_{k+1}$

Else if $\left(s \notin L_{k}\right)$ then insert $c$ into $L_{k+1}$ 


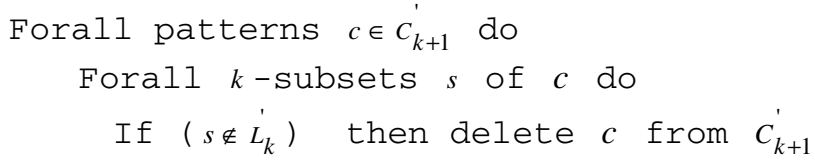

\section{Experiments}

In this section, we report our experimental results. All experiments are performed on two kinds of datasets: 1. a dense dataset, Mushroom characteristic dataset, which consists of 8,124 transactions, each with an average length of 23 items. 2. a sparse dataset, Traditional Chinese Medicine (TCM) formula dataset, which consists of 4,643 formulas with 21689 kinds of medicine involved, each with an average length of 10 kinds of medicine. TCM formula dataset is obtained from Information Institute of China Academy of Traditional Chinese Medicine.

Table 2. Correlation confidence and chi-squared value of item pairs

\begin{tabular}{|c|c|}
\hline Mushroom dataset & TCM formula dataset \\
\hline $3<->39: 0.0207035 \ldots 26.5377$ & $55<->703: 0.210326 \ldots 12.0025$ \\
\hline $9<->91: 0.0112898 \ldots 111.029$ & $55<->1187: 0.250133 \ldots .73 .2896$ \\
\hline $34<->91: 0.0120186 \ldots .7111 .33$ & $55<->3442: 0.254997 \ldots 15.7207$ \\
\hline $54<->76: 0.0117676 \ldots 7.36221$ & $452<->1187: 0.246849 \ldots 118.593$ \\
\hline
\end{tabular}

Table 2 shows the chi-squared value and the corr-confidence of partly item pairs in mushroom dataset and TCM formula dataset. For example, $3<->39$ is an item pair. The number after ":" indicates the corr-confidence and the chi-squared value respectively. In mushroom database, item pair 34<->91 has chi-squared value 7111.33 and corr-confidence 0.0120186 . Item pair $3<->39$ has chi-squared value 26.5377 and corrconfidence 0.0207035 . Although the chi-squared value of item pair $34<->91$ is very high, the corr-confidence of item pair $34<->91$ is lower than the one of item pair $3<-$ $>39$. Therefore, item pair $3<->39$ has a higher level dependence than item pair $34<-$ $>91$ according to the corr-confidence. The chi-squared statistic simultaneously and uniformly takes into account all possible combinations of the presence and absence of the various attributes being examined as a group, so when only the presence of items in patterns is concerned, the high chi-squared value cannot infer that items in patterns are highly dependent as shown in Table 2 .

Figure 1 (A) shows the runtime with limit the length of patterns and without limit the length of patterns as the minimum support ascends. Figure 1 (B) shows the runtimes with limit the length of patterns and without limit the length of patterns as the minimum all-confidence ascends with a fixed minimum support $1 \%$. When the length of patterns produced exceeds 5, almost all frequent associated patterns are correlated patterns because of the upward closure property of correlated patterns. Therefore, we put a limit to the maximal length of patterns generated. Figure 1 indicates that if the maximum length of patterns produced does not exceed 5, the runtime decreases sharply even if the minimum support or the minimum all-confidence is low. 
Table 3. Num. of patterns in mushroom data (min_sup 1\%, min_len 2, max_len 5, c_conf 1\%)

\begin{tabular}{|l|c|c|c|c|c|c|c|}
\hline All_conf (\%) & 30 & 40 & 50 & 60 & 70 & 80 & 90 \\
\hline Independent & 112 & 90 & 61 & 31 & 12 & 12 & 7 \\
\hline Associated-correlated & 3678 & 1012 & 279 & 83 & 36 & 16 & 8 \\
\hline
\end{tabular}

Table 4. Num. of patterns in TCM data (min_sup 1\%, min_len2 max_len5, all_conf 10\%)

\begin{tabular}{|l|c|c|c|c|c|c|c|c|}
\hline Corr_conf $(\%)$ & 5 & 10 & 15 & 20 & 25 & 30 & 35 & 40 \\
\hline Independent & 3 & 7 & 16 & 31 & 55 & 76 & 112 & 160 \\
\hline Associated-correlated & 1058 & 1054 & 1045 & 1030 & 1006 & 985 & 949 & 901 \\
\hline
\end{tabular}

Table 5. Num. in mushroom data (min_sup1\%, min_len 2, max_len 5, all_conf 30\%)

\begin{tabular}{|l|c|c|c|c|c|}
\hline Corr_conf $(\%)$ & 1 & 2 & 3 & 4 & 5 \\
\hline Independent & 112 & 324 & 474 & 541 & 603 \\
\hline Associated-correlated & 3678 & 3466 & 3316 & 3249 & 3187 \\
\hline
\end{tabular}

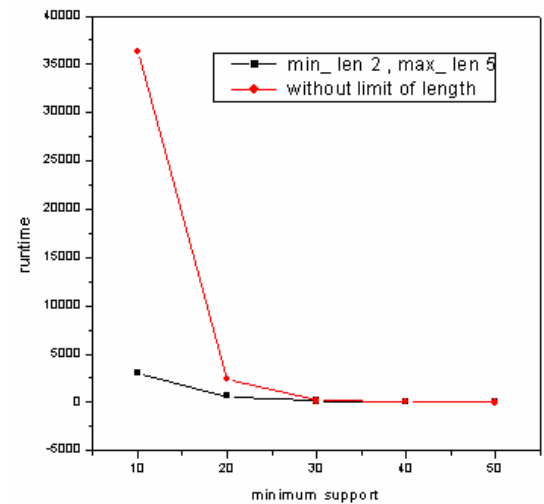

(A)

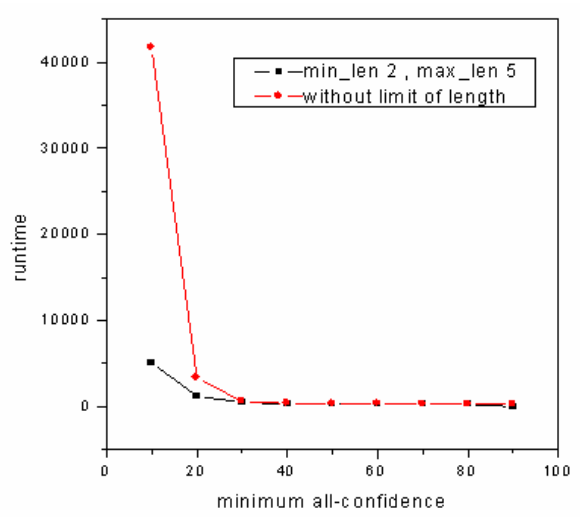

(B)

Fig. 1. The runtime of mushroom dataset

Table 3 shows the number of associated-correlated patterns and associated but not correlated patterns generated in mushroom dataset when the minimum all-confidence increases with the fixed minimum support $1 \%$, minimum corr-confidence $1 \%$, minimum pattern length 2 and maximum pattern length 5 . From Table 3, we can see that for the minimum corr-confidence $1 \%$ and the minimum all-confidence $90 \%$, there are seven associated but not correlated patterns and eight associated-correlated patterns in mushroom dataset. We can conclude that not all associated patterns are correlated even if the minimum all-confidence is much high. Table 4 and Table 5 show the number of associated-correlated patterns and associated but not correlated patterns generated in TCM dataset and mushroom dataset respectively as the 
minimum corr-confidence varies. To our surprise, when the minimum corr-confidence is $5 \%$, there are only $0.28 \%$ associated but not correlated patterns of all associated patterns in TCM dataset, while there are $16 \%$ associated but not correlated patterns of all associated patterns in mushroom dataset.

\section{Conclusions}

The mining combined association with correlation can discover both associated and correlated patterns that are extraordinary useful for making business decisions. In this paper, a new interestingness measure for correlation mining is proposed, which is not only suitable for mining long correlated patterns, but also more rational and easier to control than the chi-squared test and other commonly used measures as shown in experimental results. And an algorithm is developed for efficiently discovering all frequent both associated and correlated patterns. Experimental results show that the techniques developed in this paper are feasible.

\section{Acknowledgments}

The work is funded by subprogram of China 973 project (NO. 2003CB317006), China NSF program (No. NSFC60503018) and a grant from education ministry of Fujian of China (No. JA04248).

\section{References}

1. Bing Liu, Wynne Hsu, Yiming Ma. Pruning and Summarizing the Discovered Association. In Proc. 1999 ACM SIGKDD Int. Conf. Knowledge Discovery in Databases (KDD'99), pp. $15-18$.

2. S. Brin, R. Motwani, C. Silverstein. Beyond Market Basket: Generalizing Association Rules to Correlations. In Proc. 1997 ACM SIGMOD Int. Conf. Management of Data (SIGMOD'97), pp. 265-276.

3. H. Liu, H. Lu, L. Feng, F. Hussain. Efficient Search of Reliable Exceptions. In Proc. Pacific-Asia Conference on Knowledge Discovery and Data Mining (PAKDD'99), pp. 194203.

4. F. Mills. Statistical Methods, Pitman, 1955.

5. E. Omiecinski. Alternative interesting measures for mining associations. IEEE Trans. Knowledge and Data Engineering, 2003(15): 57-69.

6. P.-N. Tan, V. Kumar, J. Srivastava. Selecting the Right Interestingness Measure for Association Patterns. In Proc. 2002 ACM SIGKDD Int. Conf. Knowledge Discovery in Databases (KDD'02), pp. 32-41.

7. G. Piatetsky-Shapiro. Discovery, Analysis and Presentation of Strong Rules. Knowledge Discovery in Databases, AAAI/MIT Press, 1991. pp. 229-248.

8. H. T. Reynolds. The Analysis of Cross-Classifications. The Free Press, New York, 1977.

9. W.-Y. Kim, Y.-K. Lee, J. Han. CCMine: Efficient Mining of Confidence-Closed Correlated Patterns. In Proc. 2004 Pacific-Asia Conf. Knowledge Discovery and Data Mining (PAKDD'04), pp. 569-579.

10. Y.-K. Lee, W.-Y. Kim, Y. D. Cai, J. Han. CoMine: Efficient Mining of Correlated Patterns. In Proc. 2003 Int. Conf. Data Mining (ICDM'03), pp. 581-584. 